\title{
含肜醚的姜黄素衍生物的合成及生物活性研究
}

\author{
陈玉王忠波张贤夏丽娟 \\ 龚华玉 赵洪菊薛 伟* \\ (贵州大学精细化工研究开发中心 绿色农药与农业生物工程国家重点实验室培育基地 \\ 教育部绿色农药与生物工程重点实验室 贵阳 550025)
}

\begin{abstract}
摘要 采用活性拼接原理, 将肜醚引入到含杂环的姜黄素衍生物 1,4-戊二烯-3-酮结构中, 合成了一系列新的 1-芳基-5杂环-1,4-戊二烯-3-酮肜醚类化合物, 其结构经 IR, ${ }^{1} \mathrm{H}$ NMR, ${ }^{13} \mathrm{C} N M R$, ESI-MS 和元素分析确认. 初步生物活性测试结 果表明: 在药剂浓度为 $500 \mu \mathrm{g} / \mathrm{mL}$ 时, 目标化合物具有一定的抗烟草病毒(TMV)活性.

关键词 姜黄素; 肜醚; 合成; 抗 TMV 活性
\end{abstract}

\section{Synthesis and Biological Activity of Oxime Ethers of Curcumin Derivatives}

\author{
Chen, Yu Wang, Zhongbo Zhang, Xian Xia, Lijuan \\ Gong, Huayu Zhao, Hongju Xue, Wei*
}

(State Key Laboratory Breeding Base of Green Pesticide and Agricultural Bioengineering, Key Laboratory of Green

Pesticide and Bioengineering of Ministry of Education, Center for Research and Development of Fine Chemicals,

Guizhou University, Guiyang 550025)

\begin{abstract}
On the principle of biologically active factor splicing, curcumin derivatives introduced active functional group oxime ethers into the structure of 1,4-pentadien-3-one. A series of new 1-phenyl-5-heterocycle-1,4-pentadien-3-one oxime ethers were synthesized, and their structures were identified by IR, ${ }^{1} \mathrm{H}$ NMR, ${ }^{13} \mathrm{C}$ NMR, ESI-MS and elemental analysis. The preliminary bioassay results indicated that target compounds showed certain antiviral activity against TMV at a concentration of $500 \mu \mathrm{g} / \mathrm{mL}$.
\end{abstract}

Keywords curcumin; oxime ether; synthesis; anti-TMV activity

姜黄素是一类生物活性广泛的天然产物, 具有抑

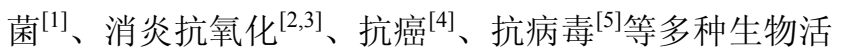
性, 结构如图 1. 姜黄素衍生物 1,5-二苯基-1,4-戊二烯-3酮类化合物同姜黄素一样有广泛的生物活性, 但其稳定 性更好，毒副作用更小. 因此，以姜黄素为先导化合物 对其进行结构修饰，期望得到更高活性的 1,4-戊二烯-3酮类化合物, 为绿色仿生农药的设计合成提供理论支 持.

肜醚类化合物有高效、低毒、低残留等优点, 且具

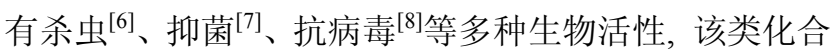
物的设计合成与活性研究引起了人们的广泛关注.<smiles>COc1cc(/C=C/C(=O)CC(=O)/C=C/c2ccc(O)c(OC)c2)ccc1O</smiles>

图 1 姜黄素的结构

Figure 1 Structure of curcumin

2008 年, 李少博等 ${ }^{[9]}$ 利用活性拼接原理, 将肜酯引 入到 1,4-戊二烯-3-酮结构中, 得到了 15 个未见文献报 道的 1,4-戊二烯-3-酮肜脂类化合物, 生物活性测试结果 表明: 化合物对小麦赤霉病菌、辣椒枯萎病菌、苹果腐

*E-mail: wxue@gzu.edu.cn

Received February 27, 2014; revised April 30, 2014; published online May 5, 2014.

Project supported by the National Tweifth Five-Year Plan for Science and Technology (No. 2011BAE06B04-09) and the Innovation Fund for Graduate Student of Guizhou University (No. 2013036). .

国家十二五科技支撑计划(No. 2011BAE06B04-09)和贵州大学研究生创新基金(No. 2013036)资助项目. 
烂病菌的抑制率为 $42 \% \sim 53 \%$, 与对照药剂恶霉灵的抑 制活性相当. 2009 年, 王振宁等 ${ }^{[10]}$ 以姜黄素为先导化合 物, 设计合成了一系列 1,5-二取代吡唑基-1,4-戊二烯-3酮类化合物, 初步生物活性结果表明: 部分化合物有一 定的抗烟草花叶病毒活性. 2010 年, 陈鹏丽 ${ }^{[11]}$ 合成了一 系列含肜醚结构的 1,4-戊二烯-3-酮类化合物, 并对化合 物进行了小菜蛾杀虫活性测试. 结果表明: 浓度为 100 $\mu \mathrm{g} / \mathrm{mL}$ 时, 化合物校正死亡率为 $68 \% \sim 79 \%$, 与阿维菌 素(校正死亡率 $82 \%$ )效果相当. 2011 年, 仇秋娟等 ${ }^{[12]}$ 采 用生物活性基团拼原理, 将肜酯引入单羰基姜黄素衍生 物 1,5-二芳基-1,4-戊二烯-3-酮结构中, 合成了 11 个不对 称 1,5-二芳基-1,4-戊二烯-3-酮肜酯类化合物，初步生物 活性测试表明: 浓度为 $0.5 \mathrm{mg} / \mathrm{L}$ 时, 所有化合物有一定 的抗黄瓜花叶病毒(CMV)活性. 2011 年, Ma 等 ${ }^{[13]}$ 合成了 1-(4-甲基芐基)哌啶-4-醞-6-氯吡啶-3-甲基肜醚. 活性测 试结果表明: 在浓度为 $1 \times 10^{-4} \mathrm{mg} / \mathrm{L}$ 时, 该化合物有很 好的抑菌活性. 本文以姜黄素为先导化合物, 采用活性 拼接原理, 将肜醚引入到含杂环的姜黄素衍生物 1,4-戊 二烯-3-酮结构中, 合成了一系列新的 1-芳基-5-杂环1,4-戊二烯-3-酮肜醚类化合物, 其结构经 IR, ${ }^{1} \mathrm{H}$ NMR, ${ }^{13} \mathrm{C}$ NMR 和 ESI-MS 确认, 并对目标化合物进行了抗烟 草花叶病毒(TMV)活性测试. 测试结果表明: 目标化合 物具有一定的抗 TMV 活性. 中间体及目标化合物的合 成路线见 Scheme 1.
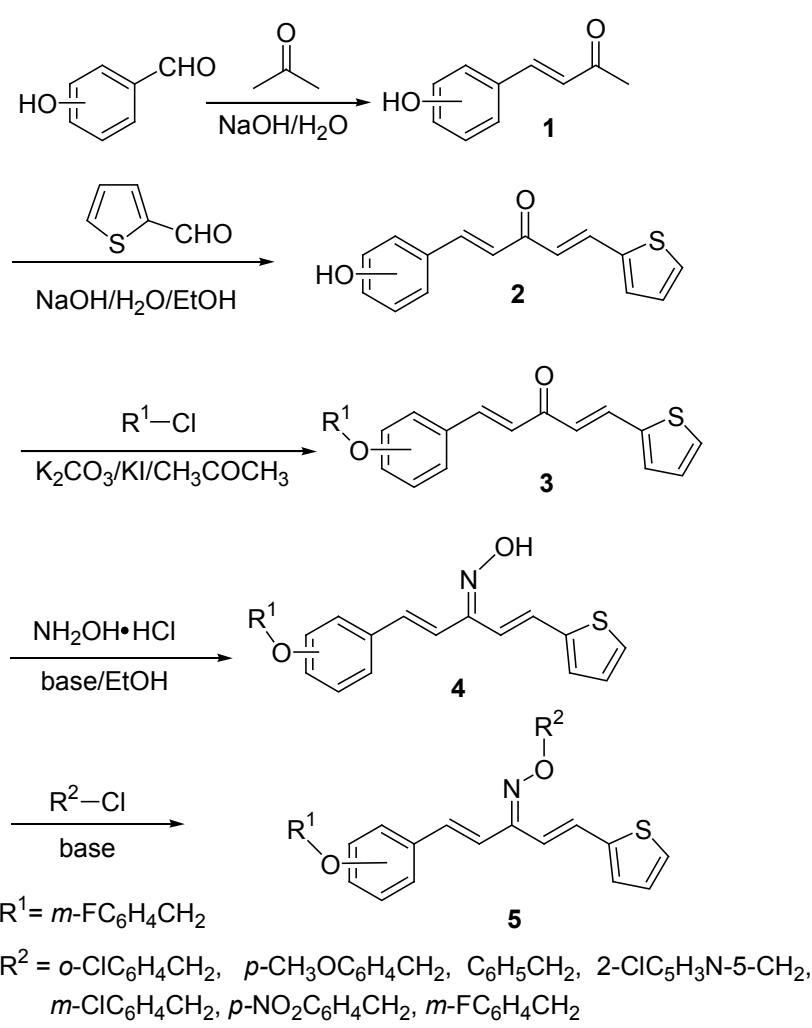

Scheme 1

\section{1 结果与讨论}

\section{1 波谱分析}

以化合物 $\mathbf{5 f}$ 为例, 对其 IR, ${ }^{1} \mathrm{H}$ NMR 和 ESI-MS 的 谱图特点进行了讨论, 结果如下.

在 IR 中, 3063, $3030 \mathrm{~cm}^{-1}$ 出现的峰是苯环碳氢伸缩 振动; $2926,2870 \mathrm{~cm}^{-1}$ 出现的峰是梄键饱和碳氢伸缩振 动; $1605 \mathrm{~cm}^{-1}$ 出现一个强尖峰, 是碳氮双键的伸缩振 动; $1508 \sim 1458 \mathrm{~cm}^{-1}$ 出现的峰是苯环的伸缩振动; 1364 $\mathrm{cm}^{-1}$ 出现的一组尖峰, 这是烯键 $\mathrm{C}-\mathrm{H}$ 的弯曲振动; $1231 \mathrm{~cm}^{-1}$ 出现弱尖峰是醚键的反对称伸缩振动; 964 $\mathrm{cm}^{-1}$ 出现的强尖峰, 是烯键(反式) $\mathrm{C}-\mathrm{H}$ 的面外弯曲振 动; $748,698 \mathrm{~cm}^{-1}$ 出现的两尖峰, 是苯环 1,3 二取代的面 外弯曲振动.

在 ${ }^{1} \mathrm{H}$ NMR 谱图中, $\delta 5.18$ 出现的双峰是由含肜醚 结构苯环上芐位的氢引起的, $\delta 5.23$ 出现单峰是含芐氧 基苯环上芐位的氢, $\delta 6.72$ 出现双峰是芐氧基苯环上 3 位氢, $\delta 6.98 \sim 7.20$ 出现多重峰是噻吩环上的 3,4 氢、芐 氧基苯环上 5 位氢、含氟苯环上的 2 位氢和烯键上的氢, $\delta 7.26 \sim 7.47$ 出现多重峰是苯环上的氢、烯键上的氢、 含氟苯环上的 4,5,6 位氢和芐氧基苯环上的 4 位氢, $\delta$ 7.26 7.47 出现多重峰是芐氧基苯环上的 6 位氢, $\delta$ $7.64 \sim 7.68$ 出现多重峰是噻吩环上的 5 位氢.

在 ESI-MS 中, $5 \mathbf{f}$ 有较强的准离子峰 $\left([\mathrm{M}+\mathrm{H}]^{+}\right)$, 表 明化合物的结构较稳定.

\section{2 合成条件优化}

以化合物 $5 \mathbf{a}$ 为例, 讨论了以不同的碱作缚酸剂, 不 同的溶剂和反应条件对 5a 产率的影响. 结果表明, 以 $\mathrm{NaH}$ 作缚酸剂, 无水 THF 为溶剂, 室温反应 $24 \mathrm{~h}$, 无新 点生成; 改成回流条件, $24 \mathrm{~h}$ 后依然无新点生成; 以 $10 \% \mathrm{NaOH}$ 水溶液作缚酸剂, 四丁基澳化铵(TBAB)为 相转移催化剂, $\mathrm{CH}_{2} \mathrm{Cl}_{2}$ 为溶剂, 回流 $3 \mathrm{~h}$, 收率为 $13 \%$; 以 $\mathrm{K}_{2} \mathrm{CO}_{3}$ 作缚酸剂, 丙酮为溶剂, 室温反应 $8 \mathrm{~h}$, 收率为 $20 \%$; 以 $\mathrm{K}_{2} \mathrm{CO}_{3}$ 作缚酸剂, 无水乙腈为溶剂, 室温反应 6 $\mathrm{h}$, 收率为 $48 \%$. 因此, 以 $\mathrm{K}_{2} \mathrm{CO}_{3}$ 作缚酸剂, 无水乙腈为 溶剂, 回流条件下, 产率较高, 为较优反应条件.

\section{3 目标化合物的抗 TMV 活性}

按照第 3.3 节的测试方法, 在药剂浓度为 $500 \mathrm{mg} / \mathrm{L}$ 时, 对目标化合物进行活体治疗、活体保护和活体针化 试验，结果如表 1 所示.

从表 1 可知: 在药剂浓度为 $500 \mathrm{mg} / \mathrm{L}$ 时，与对照药 剂宁南霉素相比, 大部分目标化合物对 TMV 有较好的 抑制活性. 治疗作用方面: 化合物 $\mathbf{5 h}, \mathbf{5 i}$ 和 $\mathbf{5 m}$ 的抑制率 分别为 $61.70 \%, 61.92 \%$ 和 $64.36 \%$ ，与宁南霉素 $(64.71 \%)$ 的效果相当; 化合物 $5 \mathbf{b}, 5 \mathbf{c}, 5 \mathrm{e}, 5 \mathbf{5}$ 和 $5 \mathbf{k}$ 的抑制率为 
表 1 化合物对 TMV 侵染的活体治疗、保护、钝化作用

Table 1 Curative, protective and inactivation effect of compounds against TMV in vivo

\begin{tabular}{lccc}
\hline 化合物 & 治疗作用 $/ \%$ & 保护作用 $/ \%$ & 针化作用 $/ \%$ \\
\hline $\mathbf{5 a}$ & 39.31 & 60.69 & 94.43 \\
$\mathbf{5 b}$ & 51.35 & 62.18 & 85.38 \\
$\mathbf{5 c}$ & 53.91 & 53.12 & 88.54 \\
$\mathbf{5 d}$ & 38.14 & 54.25 & 85.46 \\
$\mathbf{5 e}$ & 55.36 & 59.30 & 89.46 \\
$\mathbf{5 f}$ & 56.36 & 63.76 & 80.48 \\
$\mathbf{5 g}$ & 23.36 & 52.56 & 79.49 \\
$\mathbf{5 h}$ & 61.70 & 63.94 & 75.80 \\
$\mathbf{5 i}$ & 61.92 & 61.55 & 77.78 \\
$\mathbf{5 j}$ & 39.28 & 59.18 & 78.45 \\
$\mathbf{5 k}$ & 50.99 & 56.33 & 85.90 \\
$\mathbf{5 l}$ & 54.74 & 65.84 & 73.84 \\
$\mathbf{5 m}$ & 64.36 & 68.07 & 78.54 \\
宁南霉素 & 64.71 & 76.09 & 96.54 \\
\hline
\end{tabular}

50\% 60\%, 活性稍低于宁南霉素. 保护作用方面：化 合物 5a, 5b, 5f, 5h, 5i, 5l 和 $5 \mathrm{~m}$ 的抑制率为 $60 \% \sim 70 \%$, 稍低于宁南霉素 $(76.09 \%)$, 其它化合物的抑制率在 $50 \% \sim 60 \%$. 钝化作用方面: 化合物 5a $\sim 5 \mathrm{e}$ 和 $5 \mathrm{k}$ 的抑制 率均大于 $85 \%$, 稍低于宁南霉素(96.54\%), 其中, 化合 物 $5 \mathrm{a}$ 的抑制率为 $94.43 \%$ ，与宁南霉素的效果相当.

通过结构与生物活性数据分析得出: 当 $R^{2}$ 为 4-硝 基苠基、2-氯吡啶-5-亚甲基时在治疗方面表现出较好的 活性; 当 $\mathrm{R}^{2}$ 为 3-氯茮基、2-氯吡啶-5-亚甲基时在保护方 面表现出较好的活性; 当 $\mathrm{R}^{2}$ 为 2-氯茮基、4-硝基苠基、 2-氯吡啶-5-亚甲基时在钝化方面表现出较好的活性.

\section{2 结论}

本文以邻(或对)羟基苯甲醛、丙酮和 2-噻吩甲醛为 起始原料, 经醚化、肟化、再次醚化合成了 13 个新的 1-芳基-5-杂环-1,4-戊二烯-3-酩肟醚类化合物. 初步生 物活性测试结果表明: 在药剂浓度为 $500 \mathrm{mg} / \mathrm{L}$ 时, 大部 分目标化合物对 TMV 有较好的治疗、保护和钝化作用. 治疗作用方面：化合物 $5 \mathbf{h}, 5 \mathbf{i}$ 和 $5 \mathrm{~m}$ 的抑制率为 $61.70 \% \sim 64.36 \%$, 与宁南霉素的效果相当; 保护作用方 面: 化合物 5a, 5b, 5f, 5h, 5i, $5 \mathrm{l}$ 和 $5 \mathrm{~m}$ 的抑制率为 $60 \%$ $70 \%$, 稍低于宁南霉素; 钝化作用方面: 化合物 $\mathbf{5 a} \sim 5 \mathbf{e}$ 和 $5 \mathbf{k}$ 的抑制率均大于 $85 \%$, 稍低于宁南霉素.

\section{3 实验部分}

\section{1 仪器与试剂}

JEOL-ECX500 型核磁共振波谱仪(TMS 为内标, 氝 代丙酥为溶剂); Agilent ESI-MSD Trap(VL)质谱仪; Shimadzu IR Prestige-21 型红外光谱测定仪( $\mathrm{KBr}$ 压片);
X-4 型数字显示显微熔点测定仪(温度计未校正). 所用 试剂均为市售分析纯.

\section{2 实验方法}

\subsection{1中间体 2 的合成}

取 $3.57 \mathrm{~g}$ 4-(2 或 4)着基苯基-3-丁烯-2-酮(22 mmol), $2.24 \mathrm{~g}$ 2-噻吩甲醛 $(20 \mathrm{mmol}), 40 \mathrm{~mL}$ 乙醇依次加入 250 $\mathrm{mL}$ 三颈瓶中, 搅拌至溶; 冰浴条件下缓慢滴加 $40 \mathrm{~mL}$ 约 $5 \%$ 氢氧化钠溶液 (50 mmol 氢氧化钠); 室温搅拌, TLC 追踪反应.反应 $16 \mathrm{~h}$ 后, 冰浴下滴加稀盐酸至溶液 颜色不再变化 $(\mathrm{pH}$ 约为 5), 有黄色沉淀生成, 静置, 抽 滤，乙醇/水重结晶，得到中间体 2 .

1-(2-羊基取代苯基)-5-(2-噻吩)-1,4-戊二烯-3-酮 (2a): 黄色晶体, 收率 $72 \%$, m.p. 153 $155{ }^{\circ} \mathrm{C}$; ESI-MS $m / z: 279.1[\mathrm{M}+\mathrm{Na}]^{+}$.

1-(4-差基取代苯基)-5-(2-噻吩)-1,4-戊二烯-3-酮 (2b): 黄色晶体, 收率 68\%, m.p. 207 209 ${ }^{\circ} \mathrm{C}$; ESI-MS $m / z: 279.1[\mathrm{M}+\mathrm{Na}]^{+}$.

\subsection{2中间体 3 的合成}

向 $100 \mathrm{~mL}$ 三颈瓶中依次加入 $1.28 \mathrm{~g}$ 中间体 $2(5$ mmol), $1.04 \mathrm{~g} \mathrm{~K}_{2} \mathrm{CO}_{3}$ (7.5 mmol), $0.25 \mathrm{~g} \mathrm{KI}(1.50 \mathrm{mmol})$, $20 \mathrm{~mL}$ 丙酮, 搅拌; 滴加含 $0.87 \mathrm{~g} \mathrm{3}$-氟苠氯 $(6.00 \mathrm{mmol})$ 的丙酮 $5 \mathrm{~mL}$, 加热回流; TLC 追踪反应[ $V$ (石油醚)： $V($ 乙酸乙酯 $)=3: 1]$, 反应结束 $4.5 \mathrm{~h}$, 冷却至室温; 过 滤，滤液旋蒸除去丙酮，乙醇重结晶，得到中间体 $\mathbf{3}$.

1-[2-(3-氟苠氧基)苯基]-5-(2-噻吩)-1,4-戊二烯-3-酮 (3a): 黄色针状晶体, 收率 79\%. m.p. $116 \sim 117{ }^{\circ} \mathrm{C} ;{ }^{1} \mathrm{H}$ NMR $\left(\mathrm{CD}_{3} \mathrm{COCD}_{3}, 500 \mathrm{MHz}\right) \delta: 5.32\left(\mathrm{~s}, 2 \mathrm{H}, \mathrm{CH}_{2}\right), 6.91$ $\left(\mathrm{d}, J=16.0 \mathrm{~Hz}, 1 \mathrm{H}, \operatorname{Ar}\left(\mathrm{OCH}_{2}\right)-\mathrm{H}\right), 7.05(\mathrm{t}, J=7.4 \mathrm{~Hz}, 1 \mathrm{H}$, $\left.\operatorname{Ar}\left(\mathrm{OCH}_{2}\right)-\mathrm{H}\right), 7.13 \sim 7.20(\mathrm{~m}, 3 \mathrm{H}, \operatorname{Ar}(\mathrm{F})-\mathrm{H}+\mathrm{C}=\mathrm{CH})$, 7.34 (s, 1H, $\left.\operatorname{Ar}\left(\mathrm{OCH}_{2}\right)-\mathrm{H}\right), 7.37$ (s, 1H, $\left.\operatorname{Ar}(\mathrm{F})-\mathrm{H}\right), 7.40 \sim$ 7.44 (m, 2H, $\operatorname{Ar}(\mathrm{F})-\mathrm{H}), 7.47 \sim 7.52$ (m, 2H, thiophene-H), $7.66\left(\mathrm{~d}, J=5.2 \mathrm{~Hz}, 1 \mathrm{H}, \operatorname{Ar}\left(\mathrm{OCH}_{2}\right)-\mathrm{H}\right), 7.81$ (dd, $J=1.7$, $8.0 \mathrm{~Hz}, 1 \mathrm{H}$, thiophene-H), 7.87 (d, $J=15.4 \mathrm{~Hz}, 1 \mathrm{H}$, $\mathrm{CH}=), 8.11(\mathrm{~d}, J=16.0 \mathrm{~Hz}, 1 \mathrm{H}, \mathrm{CH}=)$; ESI-MS $\mathrm{m} / \mathrm{z}$ : $387.2[\mathrm{M}+\mathrm{Na}]^{+}$.

1-[4-(3-氟苠氧基)苯基]-5-(2-噻吩)-1,4-戊二烯-3-酮 (3b): 黄色片状晶体, 收率 82\%. m.p. 122 $124{ }^{\circ} \mathrm{C} ;{ }^{1} \mathrm{H}$ NMR $\left(\mathrm{CD}_{3} \mathrm{COCD}_{3}, 500 \mathrm{MHz}\right) \delta: 5.23\left(\mathrm{~s}, 2 \mathrm{H}, \mathrm{CH}_{2}\right), 6.99$ $(\mathrm{d}, J=16.0 \mathrm{~Hz}, 1 \mathrm{H}, \operatorname{Ar}(\mathrm{F})-\mathrm{H}), 7.11(\mathrm{~d}, J=8.6 \mathrm{~Hz}, 3 \mathrm{H}$, $\left.\operatorname{Ar}(\mathrm{F})-\mathrm{H}+\operatorname{Ar}\left(\mathrm{OCH}_{2}\right)-\mathrm{H}\right), 7.15 \sim 7.19(\mathrm{~m}, 2 \mathrm{H}, \mathrm{C}=\mathrm{CH})$, 7.28 (d, $J=9.8 \mathrm{~Hz}, 1 \mathrm{H}, \operatorname{Ar}(\mathrm{F})-\mathrm{H}), 7.33$ (d, $J=1.7,8.0 \mathrm{~Hz}$, 1H, $\operatorname{Ar}(\mathrm{F})-\mathrm{H}), 7.45$ (q, $J=7.2 \mathrm{~Hz}, 1 \mathrm{H}$, thiophene-H), 7.51 (s, 1H, thiophene-H), $7.64(\mathrm{~d}, J=4.6 \mathrm{~Hz}, 1 \mathrm{H}, \mathrm{CH}=), 7.72$ (t, $J=8.6 \mathrm{~Hz}, 3 \mathrm{H}$, thiophene-H+Ar(OCH$)-\mathrm{H}), 7.92(\mathrm{~d}$, $J=15.4 \mathrm{~Hz}, 1 \mathrm{H}, \mathrm{CH}=)$; ESI-MS $m / z: 387.2[\mathrm{M}+\mathrm{Na}]^{+}$. 


\subsection{3中间体 4 的合成}

向 $100 \mathrm{~mL}$ 三颈瓶中依次加入 $3.64 \mathrm{~g}$ 中间体 3 (10 $\mathrm{mmol}), 1.39 \mathrm{~g}$ 盐酸羟胺 $(20 \mathrm{mmol}), 40 \mathrm{~mL}$ 乙醇, 搅拌下 滴加 $20 \mathrm{~mL}$ 吡定, 室温摚拌 $48 \mathrm{~h}$, TLC 追踪反应[ $V$ (石油 醚) $: V$ (乙酸乙酯) $: V\left(\mathrm{CH}_{2} \mathrm{Cl}_{2}\right)=2: 1: 1$ ], 得到黄色清 夜. 旋蒸除去溶剂, 加入 $20 \mathrm{~mL} \mathrm{CH} \mathrm{Cl}_{2}$, 稀盐酸调 $\mathrm{pH}$ 约 为 5 , 水洗六次, 无水硫酸钠干燥; 过滤, 浓缩, 柱层析 分离提纯，得到中间体 4 .

1-[2-(3-氟苠氧基)苯基]-5-(2-噻吩)-1,4-戊二烯-3-酮 肜(4a): 白色粉末, 收率 50\%. m.p. $125 \sim 126{ }^{\circ} \mathrm{C} ;{ }^{1} \mathrm{H}$ $\mathrm{NMR}\left(\mathrm{CD}_{3} \mathrm{COCD}_{3}, 500 \mathrm{MHz}\right) \delta: 5.23(\mathrm{~d}, J=13.2 \mathrm{~Hz}, 2 \mathrm{H}$, $\left.\mathrm{CH}_{2}\right), 6.76(\mathrm{~d}, J=16.0 \mathrm{~Hz}, 1 \mathrm{H}, \operatorname{Ar}(\mathrm{F})-\mathrm{H}), 7.00 \sim 7.15(\mathrm{~m}$, $\left.5 \mathrm{H}, \operatorname{Ar}(\mathrm{F})-\mathrm{H}+\mathrm{Ar}\left(\mathrm{OCH}_{2}\right)-\mathrm{H}+\mathrm{C}=\mathrm{CH}\right), 7.21 \sim 7.49(\mathrm{~m}$, $7 \mathrm{H}, \quad \operatorname{Ar}(\mathrm{F})-\mathrm{H}+\mathrm{CH}=+\operatorname{Ar}\left(\mathrm{OCH}_{2}\right)-\mathrm{H}+$ thiophene- $\left.\mathrm{H}\right)$, $7.55 \sim 7.61\left(\mathrm{~m}, 1 \mathrm{H}, \operatorname{Ar}\left(\mathrm{OCH}_{2}\right)-\mathrm{H}\right), 7.70 \sim 7.74(\mathrm{~m}, 1 \mathrm{H}$, thiophene-H), 10.63 (s, $1 \mathrm{H})$; ESI-MS $m / z: 380.2[\mathrm{M}+\mathrm{H}]^{+}$, $402.2[\mathrm{M}+\mathrm{Na}]^{+}$.

1-[4-(3-氟苠氧基)苯基]-5-(2-噻吩)-1,4-戊二烯-3-酤 肜(4b): 淡黄色粉末, 收率 51\%. m.p. 131 133 ${ }^{\circ} \mathrm{C} ;{ }^{1} \mathrm{H}$ $\mathrm{NMR}\left(\mathrm{CD}_{3} \mathrm{COCD}_{3}, 500 \mathrm{MHz}\right) \delta: 5.22(\mathrm{~d}, J=7.4 \mathrm{~Hz}, 2 \mathrm{H}$, $\left.\mathrm{CH}_{2}\right), 6.75 \sim 6.91(\mathrm{~m}, 1 \mathrm{H}, \operatorname{Ar}(\mathrm{F})-\mathrm{H}), 7.04 \sim 7.19(\mathrm{~m}, 5 \mathrm{H}$, $\mathrm{C}=\mathrm{CH}+$ thiophene-H$\left.+\mathrm{Ar}\left(\mathrm{OCH}_{2}\right)-\mathrm{H}\right), 7.22 \sim 7.34(\mathrm{~m}$, $4 \mathrm{H}$, thiophene- $\mathrm{H}+\mathrm{CH}=+\operatorname{Ar}(\mathrm{F})-\mathrm{H}), 7.42 \sim 7.50(\mathrm{~m}, 3 \mathrm{H}$, $\operatorname{Ar}(\mathrm{F})-\mathrm{H}+$ thiophene-H), $7.55 \sim 7.62\left(\mathrm{~m}, 2 \mathrm{H}, \operatorname{Ar}\left(\mathrm{OCH}_{2}\right)-\right.$ $\mathrm{H}), 10.55$ (s, 1H, NOH); ESI-MS $m / z: 380.2[\mathrm{M}+\mathrm{H}]^{+}$, $402.2[\mathrm{M}+\mathrm{Na}]^{+}$.

3.2.4 目标化合物 1-芳基-5-杂环-1,4-戊二烯-3-酮肜 醚 $(5)$ 的合成

向 $50 \mathrm{~mL}$ 三颈瓶中依次加入 $0.38 \mathrm{~g}$ 中间体 4 (1 $\mathrm{mmol}), 0.28 \mathrm{~g}$ 无水 $\mathrm{K}_{2} \mathrm{CO}_{3}(2 \mathrm{mmol}), 10 \mathrm{~mL}$ 无水乙腈, 搅 拌下滴加含 $1.2 \mathrm{mmol}$ 取代苠氯的无水乙腈 $4 \mathrm{~mL}$, 升温 至回流; TLC 追踪反应 $[V$ (石油醚) $: V$ (乙酸乙酯 $)=4$ : $1]$, 反应结束后, 冷却至室温, 过滤, 浓缩滤液, 得到油 状物, 加入 $10 \mathrm{mLCH}_{2} \mathrm{Cl}_{2}$, 饱和食盐水洗涤 $(25 \mathrm{~mL} \times 4)$, 无水硫酸钠干燥, 过滤, 浓缩, 柱层析分离提纯, 得到 目标化合物 5 .

2-氯苠基-1-[2-(3-氟苠氧基)苯基]-5-(2-噻吩)-1,4-戊 二烯-3-酮肟醚 $(\mathbf{5 a})$ : 棕色粘稠物, 收率 $48 \%$. ${ }^{1} \mathrm{H} \mathrm{NMR}$ $\left(\mathrm{CD}_{3} \mathrm{COCD}_{3}, 500 \mathrm{MHz}\right) \delta: 5.21\left(\mathrm{~d}, J=8.6 \mathrm{~Hz}, 2 \mathrm{H}, \mathrm{CH}_{2}\right)$, $5.34\left(\mathrm{~d}, J=6.3 \mathrm{~Hz}, 2 \mathrm{H}, \mathrm{CH}_{2}\right), 6.72(\mathrm{~d}, J=16.0 \mathrm{~Hz}, 1 \mathrm{H}$, $\left.\mathrm{Ar}\left(\mathrm{OCH}_{2}\right)-\mathrm{H}\right), 6.98 \sim 7.18\left(\mathrm{~m}, 5 \mathrm{H}, \mathrm{Ar}(\mathrm{F})-\mathrm{H}+\mathrm{Ar}\left(\mathrm{OCH}_{2}\right)-\right.$ $\mathrm{H}+$ thiophene- $\mathrm{H}+\mathrm{C}=\mathrm{CH}), 7.21 \sim 7.53(\mathrm{~m}, 11 \mathrm{H}$, thiophene- $\left.\mathrm{H}+\mathrm{CH}=+\operatorname{Ar}(\mathrm{F})-\mathrm{H}+\mathrm{Ar}(\mathrm{Cl})-\mathrm{H}+\mathrm{Ar}\left(\mathrm{OCH}_{2}\right)-\mathrm{H}\right)$, $7.59 \sim 7.69\left(\mathrm{~m}, 2 \mathrm{H}, \mathrm{Ar}\left(\mathrm{OCH}_{2}\right)-\mathrm{H}+\mathrm{Ar}(\mathrm{Cl})-\mathrm{H}\right) ;{ }^{13} \mathrm{C} \mathrm{NMR}$ $\left(125 \mathrm{MHz}, \mathrm{CD}_{3} \mathrm{COCD}_{3}\right) \delta: 156.6,156.3,154.4,154.1$,
$141.7,135.9,132.5,130.4,130.1,129.9,129.3,128.0$, $127.6,127.1,126.0,125.7,125.4,123.2,122.0,121.4$, $117.5,116.0,114.6,114.2,112.8,77.4,69.3$; IR (KBr) $v$ : 1616, 1595, 1489, 1449, 1362, 1240, 1034, 1016, 962, 750, $698 \mathrm{~cm}^{-1}$; ESI-MS m/z: $504.2[\mathrm{M}+\mathrm{H}]^{+}$. Anal. calcd for $\mathrm{C}_{29} \mathrm{H}_{23} \mathrm{ClFNO}_{2} \mathrm{~S}$ : C 69.11, H 4.60, N 2.78; found C 69.04, $\mathrm{H} 4.76, \mathrm{~N} 2.89$.

2,4-二氯苄基-1-[2-(3-氟芐氧基)苯基]-5-(2-噻 吩)-1,4-戊二烯-3-酮肟醚 $(\mathbf{5 b})$ : 黄色晶体，收率 $61 \%$, m.p. $129 \sim 131{ }^{\circ} \mathrm{C} ;{ }^{1} \mathrm{H}$ NMR $\left(\mathrm{CD}_{3} \mathrm{COCD}_{3}, 500 \mathrm{MHz}\right) \delta$ : $5.26\left(\mathrm{~s}, 2 \mathrm{H}, \mathrm{CH}_{2}\right), 5.30\left(\mathrm{~s}, 2 \mathrm{H}, \mathrm{CH}_{2}\right), 6.71(\mathrm{~d}, J=16.0 \mathrm{~Hz}$, $\left.1 \mathrm{H}, \operatorname{Ar}\left(\mathrm{OCH}_{2}\right)-\mathrm{H}\right), 7.02 \sim 7.07\left(\mathrm{~m}, 3 \mathrm{H}, \operatorname{Ar}\left(\mathrm{OCH}_{2}\right)-\mathrm{H}+\right.$ thiophene-H+Ar(F)-H), $7.16 \sim 7.20(\mathrm{~m}, 2 \mathrm{H}, \mathrm{C}=\mathrm{CH})$, $7.28 \sim 7.30(\mathrm{~d}, J=9.7 \mathrm{~Hz}, 1 \mathrm{H}$, thiophene-H), $7.35 \sim 7.46$ $\left(\mathrm{m}, 7 \mathrm{H}, \operatorname{Ar}\left(\mathrm{OCH}_{2}\right)-\mathrm{H}+\mathrm{Ar}(\mathrm{Cl})-\mathrm{H}+\mathrm{Ar}(\mathrm{F})-\mathrm{H}+\mathrm{CH}=\right)$, $7.50 \sim 7.52\left(\mathrm{~m}, 2 \mathrm{H}, \operatorname{Ar}(\mathrm{F})-\mathrm{H}+\mathrm{Ar}\left(\mathrm{OCH}_{2}\right)-\mathrm{H}\right), 7.61 \sim 7.65$ (d, $J=17.2 \mathrm{~Hz}, 1 \mathrm{H}$, thiophene-H), $7.70(\mathrm{~d}, J=6.3 \mathrm{~Hz}, 1 \mathrm{H}$, $\mathrm{Ar}(\mathrm{Cl})-\mathrm{H}) ;{ }^{13} \mathrm{C}$ NMR (125 MHz, $\left.\mathrm{CD}_{3} \mathrm{COCD}_{3}\right) \delta: 156.7$, $154.6,141.7,140.2,135.1,133.7,133.6,132.7,131.1$, $130.5,128.9,128.2,128.0,127.7,126.1,125.3,123.2$, $121.3,117.4,114.7,114.5,114.2,114.1,112.9,72.7$, 69.3; IR (KBr) v: 1653, 1616, 1589, 1558, 1489, 1450, 1381, 1314, 1250, 1109, 1072, 1043, 961, 908, 854, 837, 814, 775, 754, 716, $679 \mathrm{~cm}^{-1}$; ESI-MS m/z: $538.2[\mathrm{M}+$ $\mathrm{H}]^{+}$. Anal. calcd. for $\mathrm{C}_{29} \mathrm{H}_{22} \mathrm{Cl}_{2} \mathrm{FNO}_{2} \mathrm{~S}: \mathrm{C} 64.69, \mathrm{H} 4.12, \mathrm{~N}$ 2.60; found C 64.15, H 4.15, N 2.71.

4-硝基苠基-1-[2-(3-氟苠氧基)苯基]-5-(2-噻吩)-1,4戊二烯-3-酮肟醚 $(5 \mathrm{c})$ : 淡黄色粉末，收率 36\%. m.p. 86 $88{ }^{\circ} \mathrm{C} ;{ }^{1} \mathrm{H}$ NMR $\left(\mathrm{CD}_{3} \mathrm{COCD}_{3}, 500 \mathrm{MHz}\right) \delta: 5.25$ (d, $J=$ $\left.12.0 \mathrm{~Hz}, 2 \mathrm{H}, \mathrm{CH}_{2}\right), 5.39\left(\mathrm{~d}, J=14.3 \mathrm{~Hz}, 2 \mathrm{H}, \mathrm{CH}_{2}\right), 6.99 \sim$ $7.21\left(\mathrm{~m}, 6 \mathrm{H}, \mathrm{Ar}(\mathrm{F})-\mathrm{H}+\mathrm{Ar}\left(\mathrm{OCH}_{2}\right)-\mathrm{H}+\right.$ thiophene- $\mathrm{H}+\mathrm{C}=$ $\mathrm{CH}), 7.27 \sim 7.38\left(\mathrm{~m}, 5 \mathrm{H}\right.$, thiophene- $\mathrm{H}+\mathrm{Ar}\left(\mathrm{OCH}_{2}\right)-\mathrm{H}+$ $\operatorname{Ar}(\mathrm{F})-\mathrm{H}), 7.44 \sim 7.61\left(\mathrm{~m}, 3 \mathrm{H}, \mathrm{CH}=+\mathrm{Ar}\left(\mathrm{OCH}_{2}\right)-\mathrm{H}\right)$, $7.65 \sim 7.72\left(\mathrm{~m}, 3 \mathrm{H}, \operatorname{Ar}\left(\mathrm{NO}_{2}\right)-\mathrm{H}+\right.$ thiophene- $\left.\mathrm{H}\right), 8.22 \sim$ $8.25\left(\mathrm{~m}, \quad 2 \mathrm{H}, \quad \operatorname{Ar}\left(\mathrm{NO}_{2}\right)-\mathrm{H}\right) ;{ }^{13} \mathrm{C} \mathrm{NMR}(125 \mathrm{MHz}$, $\left.\mathrm{CD}_{3} \mathrm{COCD}_{3}\right) \delta: 156.3,154.3,146.4,141.6,132.8,130.6$, $130.5,129.9,129.5,128.6,128.5,128.4,128.2,127.8$, $127.2,126.1,125.6,123.5,121.8,121.3,117.5,115.9$, $114.6,114.5,114.2,114.0,112.8,74.8,69.3$; IR (KBr) $v$ : 1601, 1558, 1516, 1489, 1450, 1344, 1240, 1067, 961, 733, $687 \mathrm{~cm}^{-1}$; ESI-MS m/z: $537.3[\mathrm{M}+\mathrm{Na}]^{+}$. Anal. calcd for $\mathrm{C}_{29} \mathrm{H}_{23} \mathrm{FN}_{2} \mathrm{O}_{4} \mathrm{~S}$ : C 67.69, H 4.51, N 5.44; found $\mathrm{C} 67.14, \mathrm{H}$ 4.47, N 5.67 .

4- 甲氧基苄基-1-[2-(3-氟苠氧基) 苯基]-5-(2-噻 吩)-1,4-戊二烯-3-酠肜醚(5d): 白色粉末, 收率 29\%. 
m.p. 93 96 ${ }^{\circ} \mathrm{C} ;{ }^{1} \mathrm{H} \mathrm{NMR}\left(\mathrm{CD}_{3} \mathrm{COCD}_{3}, 500 \mathrm{MHz}\right) \delta: 3.79$ (s, $\left.3 \mathrm{H}, \mathrm{OCH}_{3}\right), 5.15$ (d, $\left.J=9.2 \mathrm{~Hz}, 2 \mathrm{H}, \mathrm{CH}_{2}\right), 5.25(\mathrm{~s}, 2 \mathrm{H}$, $\left.\mathrm{CH}_{2}\right), 6.91 \sim 6.92\left(\mathrm{~m}, 2 \mathrm{H}, \operatorname{Ar}\left(\mathrm{OCH}_{2}\right)-\mathrm{H}+\operatorname{Ar}(\mathrm{F})-\mathrm{H}\right), 7.00 \sim$ $7.04\left(\mathrm{~m}, \quad 2 \mathrm{H}, \quad \operatorname{Ar}\left(\mathrm{OCH}_{3}\right)-\mathrm{H}\right), \quad 7.07 \sim 7.10(\mathrm{~m}, 2 \mathrm{H}$, $\left.\operatorname{Ar}\left(\mathrm{OCH}_{3}\right)-\mathrm{H}\right), 7.14\left(\mathrm{~d}, J=8.0 \mathrm{~Hz}, 2 \mathrm{H}, \operatorname{Ar}\left(\mathrm{OCH}_{2}\right)-\mathrm{H}+\right.$ thiophene-H), $7.22(\mathrm{~d}, J=3.4 \mathrm{~Hz}, 1 \mathrm{H}, \mathrm{C}=\mathrm{CH}), 7.30 \sim$ $7.44\left(\mathrm{~m}, 7 \mathrm{H}\right.$, thiophene- $\mathrm{H}+\mathrm{C}=\mathrm{CH}+\mathrm{Ar}\left(\mathrm{OCH}_{2}\right)-\mathrm{H}+$ $\mathrm{CH}=+\operatorname{Ar}(\mathrm{F})-\mathrm{H}), 7.50(\mathrm{~d}, J=4.6 \mathrm{~Hz}, 1 \mathrm{H}, \operatorname{Ar}(\mathrm{F})-\mathrm{H}), 7.60$ (d, $\left.J=16.6 \mathrm{~Hz}, 1 \mathrm{H}, \operatorname{Ar}\left(\mathrm{OCH}_{2}\right)-\mathrm{H}\right), 7.72(\mathrm{~d}, J=6.3 \mathrm{~Hz}, 1 \mathrm{H}$, thiophene-H); ${ }^{13} \mathrm{C}$ NMR $\left(125 \mathrm{MHz}, \mathrm{CD}_{3} \mathrm{COCD}_{3}\right) \delta: 159.7$, $156.3,153.1,141.7,130.5,130.1,129.9,129.7,129.3$, $128.7,128.1,127.1,126.9,125.8,123.2,122.2,121.3$, $116.2,114.6,114.5,114.2,114.0,113.7,112.8,76.2$, 69.3,54.7; IR (KBr) v: 2934, 2835, 1616, 1595, 1516, 1489, 1448, 1371, 1302, 1254, 1238, 1109, 1015, 953, 779, 750, $706 \mathrm{~cm}^{-1}$; ESI-MS $m / z: 500.3[\mathrm{M}+\mathrm{H}]^{+}, 522.3[\mathrm{M}+$ $\mathrm{Na}]^{+}$. Anal. calcd for $\mathrm{C}_{30} \mathrm{H}_{26} \mathrm{FNO}_{3} \mathrm{~S}: \mathrm{C} 72.12, \mathrm{H} 5.25, \mathrm{~N}$, 2.80; found C 72.50, H 5.56, N 2.99.

2-氯-5-甲基吡啶-1-[2-(3-氟芐氧基)苯基]-5-(2-噻 吩)-1,4-戊二烯-3-酮肜醚(5e): 黄色油状物, 收率 46\%. ${ }^{1} \mathrm{H}$ NMR $\left(\mathrm{CD}_{3} \mathrm{COCD}_{3}, 500 \mathrm{MHz}\right) \delta: 5.26\left(\mathrm{~s}, 2 \mathrm{H}, \mathrm{CH}_{2}\right)$, $5.33\left(\mathrm{~d}, J=6.8 \mathrm{~Hz}, 2 \mathrm{H}, \mathrm{CH}_{2}\right), 6.78(\mathrm{~d}, J=16.0 \mathrm{~Hz}, 1 \mathrm{H}$, $\left.\operatorname{Ar}\left(\mathrm{OCH}_{2}\right)-\mathrm{H}\right), 7.06 \sim 7.23\left(\mathrm{~m}, 5 \mathrm{H}, \operatorname{Ar}(\mathrm{F})-\mathrm{H}+\operatorname{Ar}\left(\mathrm{OCH}_{2}\right)-\right.$ $\mathrm{H}+$ thiophene- $\mathrm{H}+\mathrm{C}=\mathrm{CH}), 7.28 \sim 7.56(\mathrm{~m}, 8 \mathrm{H}$, thiophene- $\mathrm{H}+\mathrm{Ar}\left(\mathrm{OCH}_{2}\right)-\mathrm{H}+$ pyridine- $\mathrm{H}+\mathrm{CH}=+$ $\operatorname{Ar}(\mathrm{F})-\mathrm{H}), 7.65 \sim 7.69\left(\mathrm{~m}, 1 \mathrm{H}, \operatorname{Ar}\left(\mathrm{OCH}_{2}\right)-\mathrm{H}\right), 7.73 \sim 7.75$ (d, $J=7.4 \mathrm{~Hz}, 1 \mathrm{H}$, thiophene-H), $7.89 \sim 7.95(\mathrm{~m}, 1 \mathrm{H}$, pyridine-H), 8.54 (d, $J=12.6 \mathrm{~Hz}, 1 \mathrm{H}$, pyridine- $\mathrm{H}) ;{ }^{13} \mathrm{C}$ NMR $\left(125 \mathrm{MHz}, \mathrm{CD}_{3} \mathrm{COCD}_{3}\right) \delta: 156.7,154.6,150.5$, $149.6,141.7,139.2,133.3,132.6,130.5,129.9,129.5$, $128.2,127.7,127.3,126.1,125.3,124.1,123.2,122.0$, 121.3, 117.4, 115.9, 114.7, 114.3, 114.0, 112.9, 72.8, 69.3; IR (KBr) v: 1616, 1591, 1489, 1458, 1387, 1240, 1105, 1016, 964, 750, $704 \mathrm{~cm}^{-1}$; ESI-MS m/z: $505.2[\mathrm{M}+\mathrm{H}]^{+}$. Anal. calcd for $\mathrm{C}_{28} \mathrm{H}_{22} \mathrm{ClFN}_{2} \mathrm{O}_{2} \mathrm{~S}$ : C 66.59, H 4.39, N 5.55; found C 66.58, H 5.09, N 5.79.

芐基-1-[2-(3-氟芐氧基)苯基]-5-(2-噻吩)-1,4-戊二 烯-3-酮肜醚(5f): 棕色粘稠物, 收率 $42 \% .{ }^{1} \mathrm{H}$ NMR $\left(\mathrm{CD}_{3} \mathrm{COCD}_{3}, 500 \mathrm{MHz}\right) \delta: 5.18\left(\mathrm{~s}, 2 \mathrm{H}, \mathrm{CH}_{2}\right), 5.23(\mathrm{~d}, J=$ $\left.6.3 \mathrm{~Hz}, 2 \mathrm{H}, \mathrm{CH}_{2}\right), 6.72\left(\mathrm{~d}, J=16.0 \mathrm{~Hz}, 1 \mathrm{H}, \operatorname{Ar}\left(\mathrm{OCH}_{2}\right)-\mathrm{H}\right)$, $6.98 \sim 7.20\left(\mathrm{~m}, 6 \mathrm{H}, \operatorname{Ar}(\mathrm{F})-\mathrm{H}+\operatorname{Ar}\left(\mathrm{OCH}_{2}\right)-\mathrm{H}+\mathrm{C}=\mathrm{CH}+\right.$ thiophene-H), $7.26 \sim 7.47\left(\mathrm{~m}, 11 \mathrm{H}, \operatorname{Ar}\left(\mathrm{OCH}_{2}\right)-\mathrm{H}+\mathrm{Ar}(\mathrm{F})-\right.$ $\mathrm{H}+\mathrm{CH}=+\mathrm{Ar}-\mathrm{H}), 7.58 \sim 7.62\left(\mathrm{~m}, 1 \mathrm{H}, \operatorname{Ar}\left(\mathrm{OCH}_{2}\right)-\mathrm{H}\right)$, $7.64 \sim 7.68\left(\mathrm{~m}, 1 \mathrm{H}\right.$, thiophene-H); ${ }^{13} \mathrm{C}$ NMR $(125 \mathrm{MHz}$, $\left.\mathrm{CD}_{3} \mathrm{COCD}_{3}\right) \delta: 156.3,153.4,141.9,141.4,138.4,132.1$,
$130.5,130.1,129.8,129.3,128.9,128.4,128.1,127.8$, $127.5,127.2,127.0,125.9,125.7,123.2,122.2,121.7$, 121.3, 117.6, 116.2, 114.3, 114.1, 112.8, 76.4, 69.3; IR (KBr) $v: 3063,3030,2926,2870,1614,1593,1498,1452$, 1240, 1015, 962, 748, $698 \mathrm{~cm}^{-1}$; ESI-MS $m / z$ : $470.2[\mathrm{M}+$ $\mathrm{H}]^{+}$. Anal. calcd for $\mathrm{C}_{29} \mathrm{H}_{24} \mathrm{FNO}_{2} \mathrm{~S}: \mathrm{C} 74.18, \mathrm{H} 5.15, \mathrm{~N}$ 2.98; found C 74.26, H 5.28, N 3.16 .

4-甲氧基苄基-1-[4-(3-氟苄氧基)苯基]-5-(2-噻 吩)-1,4-戊二烯-3-酮肟醚 $(\mathbf{5 g})$ : 黄色粘稠物, 收率 54\%. ${ }^{1} \mathrm{H}$ NMR $\left(\mathrm{CD}_{3} \mathrm{COCD}_{3}, 500 \mathrm{MHz}\right) \delta: 3.76\left(\mathrm{~s}, 3 \mathrm{H}, \mathrm{OCH}_{3}\right)$, $5.13\left(\mathrm{~s}, 2 \mathrm{H}, \mathrm{CH}_{2}\right), 5.18\left(\mathrm{~s}, 2 \mathrm{H}, \mathrm{CH}_{2}\right), 6.75 \sim 6.92(\mathrm{~m}, 3 \mathrm{H}$, $\left.\operatorname{Ar}(\mathrm{F})-\mathrm{H}+\operatorname{Ar}\left(\mathrm{OCH}_{2}\right)-\mathrm{H}\right), 7.00 \sim 7.10\left(\mathrm{~m}, 4 \mathrm{H}, \operatorname{Ar}\left(\mathrm{OCH}_{3}\right)-\right.$ $\mathrm{H}), 7.14 \sim 7.29(\mathrm{~m}, 5 \mathrm{H}$, thiophene- $\mathrm{H}+\mathrm{C}=\mathrm{CH}+\operatorname{Ar}(\mathrm{F})-\mathrm{H})$, $7.35 \sim 7.47(\mathrm{~m}, 5 \mathrm{H}, \mathrm{CH}=+\mathrm{Ar}(\mathrm{F})-\mathrm{H}+$ thiophene-H), 7.52 (t, $\left.J=7.7 \mathrm{~Hz}, 2 \mathrm{H}, \operatorname{Ar}\left(\mathrm{OCH}_{2}\right)-\mathrm{H}\right) ;{ }^{13} \mathrm{C}$ NMR $(125 \mathrm{MHz}$, $\left.\mathrm{CD}_{3} \mathrm{COCD}_{3}\right) \delta: 159.6,159.4,153.2,142.0,141.9,136.5$, $133.5,130.4,130.0,129.5,129.2,128.9,128.5,128.1$, $127.9,126.9,125.8,123.2,121.7,119.8,116.4,115.2$, $114.6,114.5,114.2,114.0,113.8,76.2,68.8,54.7$; IR (KBr) v: 2928, 1603, 1508, 1449, 1302, 1244, 1175, 1144, $1018,1011,955,829,781 \mathrm{~cm}^{-1}$; ESI-MS $m / z: 500.3[\mathrm{M}+$ $\mathrm{H}]^{+}$. Anal. calcd for $\mathrm{C}_{30} \mathrm{H}_{26} \mathrm{FNO}_{3} \mathrm{~S}: \mathrm{C} 72.12, \mathrm{H} 5.25, \mathrm{~N}$ 2.80; found C 72.43, H 5.29, N 2.86.

2-氯-5-甲基吡啶-1-[4-(3-氟芐氧基)苯基]-5-(2-噻 吩)-1,4-戊二烯-3-酮肜醚(5h): 棕色粘稠物, 收率 52\%. ${ }^{1} \mathrm{H}$ NMR $\left(\mathrm{CD}_{3} \mathrm{COCD}_{3}, 500 \mathrm{MHz}\right) \delta: 5.19\left(\mathrm{~s}, 2 \mathrm{H}, \mathrm{CH}_{2}\right)$, 5.27 (s, 2H, $\left.\mathrm{CH}_{2}\right), 6.73(\mathrm{~d}, J=16.0 \mathrm{~Hz}, 1 \mathrm{H}, \operatorname{Ar}(\mathrm{F})-\mathrm{H})$, $7.04 \sim 7.11\left(\mathrm{~m}, 4 \mathrm{H}, \operatorname{Ar}\left(\mathrm{OCH}_{2}\right)-\mathrm{H}+\right.$ thiophene-H), $7.22 \sim$ $7.45(\mathrm{~m}, 9 \mathrm{H}, \quad \mathrm{CH}=\mathrm{CH}+$ pyridine- $\mathrm{H}+\operatorname{Ar}(\mathrm{F})-\mathrm{H}+$ thiophene-H), $7.58\left(\mathrm{~d}, J=9.2 \mathrm{~Hz}, 2 \mathrm{H}, \operatorname{Ar}\left(\mathrm{OCH}_{2}\right)-\mathrm{H}\right), 7.90$ (d, $J=8.0 \mathrm{~Hz}, 1 \mathrm{H}$, pyridine- $\mathrm{H}), 8.48$ (s, $1 \mathrm{H}$, pyridine- $\mathrm{H}$ ); ${ }^{13} \mathrm{C}$ NMR $\left(125 \mathrm{MHz}, \mathrm{CD}_{3} \mathrm{COCD}_{3}\right) \delta: 159.5,154.2,150.4$, $149.6,141.8,140.2,139.3,137.1,133.4,130.4,129.4$, $129.0,128.2,128.0,127.5,126.0,124.1,123.2,121.1$, $115.3,114.8,114.6,114.5,114.2,114.0,72.8,68.8$; IR (KBr) $v$ : 2928, 1603, 1591, 1508, 1458, 1383, 1246, 1175, 1105, 1016, 964, 824, 781, $704 \mathrm{~cm}^{-1}$; ESI-MS m/z: 505.2 $[\mathrm{M}+\mathrm{H}]^{+}$. Anal. calcd for $\mathrm{C}_{28} \mathrm{H}_{22} \mathrm{ClFN}_{2} \mathrm{O}_{2} \mathrm{~S}: \mathrm{C} 66.59, \mathrm{H}$ 4.39, N 5.55; found C 66.57, H 4.64, N 5.50.

4-硝基芐基-1-[4-(3-氟芐氧基)苯基]-5-(2-噻吩)-1,4戊二烯-3-酮肜醚(5i): 黄色粘稠物, 收率 38\%. ${ }^{1} \mathrm{H}$ NMR $\left(\mathrm{CD}_{3} \mathrm{COCD}_{3}, 500 \mathrm{MHz}\right) \delta: 5.20\left(\mathrm{~s}, 2 \mathrm{H}, \mathrm{CH}_{2}\right), 5.36(\mathrm{~d}, J=$ $\left.39.5 \mathrm{~Hz}, 2 \mathrm{H}, \mathrm{CH}_{2}\right), 6.89 \sim 6.85(\mathrm{~m}, 1 \mathrm{H}, \mathrm{Ar}(\mathrm{F})-\mathrm{H}), 7.28 \sim$ $7.04\left(\mathrm{~m}, \quad 7 \mathrm{H}, \quad \mathrm{C}=\mathrm{CH}+\operatorname{Ar}\left(\mathrm{OCH}_{2}\right)-\mathrm{H}+\operatorname{Ar}(\mathrm{F})-\mathrm{H}+\right.$ thiophene-H), $7.47 \sim 7.41(\mathrm{~m}, 2 \mathrm{H}, \mathrm{CH}=), 7.57 \sim 7.52(\mathrm{~m}$, 
$5 \mathrm{H}, \quad \operatorname{Ar}\left(\mathrm{OCH}_{2}\right)-\mathrm{H}+\operatorname{Ar}\left(\mathrm{NO}_{2}\right)-\mathrm{H}+$ thiophene- $\left.\mathrm{H}\right), 7.73$ (t, $\left.J=7.7 \mathrm{~Hz}, \quad 1 \mathrm{H}, \quad \operatorname{Ar}\left(\mathrm{NO}_{2}\right)-\mathrm{H}\right), 8.31 \sim 8.25(\mathrm{~m}, 1 \mathrm{H}$, $\left.\operatorname{Ar}\left(\mathrm{NO}_{2}\right)-\mathrm{H}\right) ;{ }^{13} \mathrm{C} \mathrm{NMR}\left(125 \mathrm{MHz}, \mathrm{CD}_{3} \mathrm{COCD}_{3}\right) \delta: 159.6$, $154.3,147.5,146.4,141.8,137.2,130.5,129.4,129.0$, $128.6,128.5,128.0,127.6,126.1,124.2,123.5,123.2$, $121.1,115.2,115.1,114.8,114.6,114.5,114.2,114.0$, 74.8, 68.8; IR (KBr) $v_{\max }: 1605,1593,1508,1450,1344$, 1260, 1240, 1175, 1072, 1016, 960, 856, 818, $689 \mathrm{~cm}^{-1}$; ESI-MS $m / z: 515.3[\mathrm{M}+\mathrm{H}]^{+}$. Anal. calcd for $\mathrm{C}_{29} \mathrm{H}_{23} \mathrm{FN}_{2} \mathrm{O}_{4} \mathrm{~S}$ : C 67.69, H 4.51, N 5.44; found $\mathrm{C} 67.34, \mathrm{H}$ $4.80, \mathrm{~N} 5.62$.

2,4-二氯苄基-1-[4-(3-氟苠氧基) 苯基]-5-(2-噻 吩)-1,4-戊二烯-3-酮肜醚(5j): 黄色油状物, 收率 47\%. ${ }^{1} \mathrm{H} \mathrm{NMR}\left(\mathrm{CD}_{3} \mathrm{COCD}_{3}, 500 \mathrm{MHz}\right) \delta: 5.20(\mathrm{~d}, J=6.3 \mathrm{~Hz}$, $\left.2 \mathrm{H}, \mathrm{CH}_{2}\right), 5.31\left(\mathrm{~s}, 2 \mathrm{H}, \mathrm{CH}_{2}\right), 6.72 \sim 6.87(\mathrm{~m}, 1 \mathrm{H}, \operatorname{Ar}(\mathrm{F})-\mathrm{H})$, $7.03 \sim 7.13\left(\mathrm{~m}, 4 \mathrm{H}, \quad \operatorname{Ar}\left(\mathrm{OCH}_{2}\right)-\mathrm{H}+\right.$ thiophene- $\mathrm{H}+\mathrm{C}=$ $\mathrm{CH}), 7.17 \sim 7.21(\mathrm{~m}, 1 \mathrm{H}, \mathrm{C}=\mathrm{CH}), 7.25 \sim 7.35(\mathrm{~m}, 4 \mathrm{H}$, thiophene-H $+\mathrm{Ar}(\mathrm{Cl})-\mathrm{H}+\mathrm{Ar}(\mathrm{F})-\mathrm{H}), 7.38 \sim 7.46(\mathrm{~m}, 3 \mathrm{H}$, $\mathrm{CH}=+\mathrm{Ar}(\mathrm{Cl})-\mathrm{H}), \quad 7.50 \sim 7.61 \quad(\mathrm{~m}, 5 \mathrm{H}, \quad \operatorname{Ar}(\mathrm{F})-\mathrm{H}+$ $\operatorname{Ar}\left(\mathrm{OCH}_{2}\right)-\mathrm{H}+$ thiophene- $\left.\mathrm{H}+\mathrm{Ar}(\mathrm{Cl})-\mathrm{H}\right) ;{ }^{13} \mathrm{C}$ NMR $(125$ $\left.\mathrm{MHz}, \mathrm{CD}_{3} \mathrm{COCD}_{3}\right) \delta: 159.1,154.2,154.1,141.7,137.2$, $135.1,134.1,133.8,131.3,130.6,130.4,129.7,129.5$, $128.9,128.6,128.1,127.9,127.5,127.3,127.1,126.0$, $123.2,121.1,119.3,116.0,115.2,115.1,114.7,114.6$, 114.4, 114.2, 114.0, 72.7, 68.8; IR (KBr) v: 3067, 3036, 2926, 2870, 1628, 1602, 1558, 1506, 1420, 1229, 1175, 1040, 821, $700 \mathrm{~cm}^{-1}$; ESI-MS m/z: $453.2[\mathrm{M}+\mathrm{H}]^{+}$. Anal. calcd for $\mathrm{C}_{29} \mathrm{H}_{22} \mathrm{Cl}_{2} \mathrm{FNO}_{2} \mathrm{~S}$ : C 64.69, $\mathrm{H} 4.12, \mathrm{~N} 2.60$; found C 64.83, H 4.06, N 2.42 .

苠基-1-[4-(3-氟苠氧基)苯基]-5-(2-噻吩)-1,4-戊二 烯-3-酮肟醚(5k): 黄色油状物, 收率 38\%. ${ }^{1} \mathrm{H}$ NMR $\left(\mathrm{CD}_{3} \mathrm{COCD}_{3}, 500 \mathrm{MHz}\right) \delta: 5.25\left(\mathrm{~s}, 2 \mathrm{H}, \mathrm{CH}_{2}\right), 5.33(\mathrm{~s}, 2 \mathrm{H}$, $\left.\mathrm{CH}_{2}\right), 6.83(\mathrm{~d}, J=16.0 \mathrm{~Hz}, 1 \mathrm{H}, \operatorname{Ar}(\mathrm{F})-\mathrm{H}), 7.11 \sim 7.19(\mathrm{~m}$, $\left.4 \mathrm{H}, \quad \operatorname{Ar}\left(\mathrm{OCH}_{2}\right)-\mathrm{H}+\mathrm{C}=\mathrm{CH}\right), 7.25 \sim 7.40(\mathrm{~m}, 6 \mathrm{H}$, thiophene-H $+\mathrm{Ar}(\mathrm{F})-\mathrm{H}+\mathrm{CH}=), 7.43 \sim 7.54(\mathrm{~m}, 7 \mathrm{H}$, thiophene-H+Ar-H+Ar(F)-H), 7.63 (d, J=9.2 Hz, 2H, $\left.\operatorname{Ar}\left(\mathrm{OCH}_{2}\right)-\mathrm{H}\right) ;{ }^{13} \mathrm{C} \mathrm{NMR}\left(125 \mathrm{MHz}, \mathrm{CD}_{3} \mathrm{COCD}_{3}\right) \delta: 159.5$, $153.4,142.0,138.4,136.7,130.4,129.5,128.9,128.4$, $128.1,128.0,127.8,127.1,125.9,123.2,121.6,115.2$, 115.0, 114.6, 114.5, 114.2, 114.0, 76.4, 68.8; IR (KBr) v: 3032, 2920, 1605, 1558, 1508, 1458, 1364, 1231, 1175, 1015, 964, $698 \mathrm{~cm}^{-1}$; ESI-MS m/z: $470.3[\mathrm{M}+\mathrm{H}]^{+}$. Anal. calcd for $\mathrm{C}_{29} \mathrm{H}_{24} \mathrm{FNO}_{2} \mathrm{~S}$ : C 74.18, H 5.15, N 2.98; found $\mathrm{C}$ 74.54, H 5.12, N 3.02 .

3-氯苄基-1-[4-(3-氟芐氧基)苯基]-5-(2-噻吩)-1,4-戊 二烯-3-酮肜醚(5l): 黄色油状物, 收率 $32 \%$. ${ }^{1} \mathrm{H}$ NMR $\left(\mathrm{CD}_{3} \mathrm{COCD}_{3}, 500 \mathrm{MHz}\right) \delta: 5.15\left(\mathrm{~d}, J=5.2 \mathrm{~Hz}, 2 \mathrm{H}, \mathrm{CH}_{2}\right)$, $5.37\left(\mathrm{~s}, 2 \mathrm{H}, \mathrm{CH}_{2}\right), 6.75 \sim 6.89(\mathrm{~m}, 1 \mathrm{H}, \mathrm{Ar}(\mathrm{F})-\mathrm{H}), 7.02 \sim$ $7.11\left(\mathrm{~m}, 4 \mathrm{H}, \operatorname{Ar}\left(\mathrm{OCH}_{2}\right)-\mathrm{H}+\mathrm{C}=\mathrm{CH}\right), 7.16 \sim 7.37(\mathrm{~m}, 7 \mathrm{H}$, thiophene-H $+\operatorname{Ar}(\mathrm{F})-\mathrm{H}+\mathrm{CH}=+\operatorname{Ar}(\mathrm{Cl})-\mathrm{H}), \quad 7.40 \sim 7.50$ $(\mathrm{m}, \quad 4 \mathrm{H}, \quad \operatorname{Ar}(\mathrm{F})-\mathrm{H}+\operatorname{Ar}(\mathrm{Cl})-\mathrm{H}), 7.53 \sim 7.58(\mathrm{~m}, 3 \mathrm{H}$, thiophene-H $\left.+\mathrm{Ar}\left(\mathrm{OCH}_{2}\right)-\mathrm{H}\right) ;{ }^{13} \mathrm{C} \quad \mathrm{NMR} \quad(125 \mathrm{MHz}$, $\left.\mathrm{CD}_{3} \mathrm{COCD}_{3}\right) \delta: 159.5,159.0,153.8,141.8,136.9,135.9$, $133.9,132.8,130.4,129.9,129.7,129.3,128.9,128.6$, $128.1,128.0,127.4,127.1,126.0,123.2,121.3,119.5$, 116.1, 115.2, 114.9, 114.6, 114.4, 114.2, 114.0, 73.4, 68.8; IR (KBr) v: 3067, 2916, 1603, 1506, 1447, 1244, 1175, 1034, 1015, 962, 822, 750, $702 \mathrm{~cm}^{-1}$; ESI-MS m/z: 504.3 $[\mathrm{M}+\mathrm{H}]^{+}$. Anal. calcd for $\mathrm{C}_{29} \mathrm{H}_{23} \mathrm{ClFNO}_{2} \mathrm{~S}$ : C $69.11, \mathrm{H}$ 4.60, N 2.78; found C 69.50, H 5.08, N 2.96.

3-氯苠基-1-[2-(3-氟苠氧基)苯基]-5-(2-噻吩)-1,4-戊 二烯-3-酮肟醚 $(\mathbf{5 m})$ : 黄色油状物, 收率 $36 \%$. ${ }^{1} \mathrm{H}$ NMR $\left(\mathrm{CD}_{3} \mathrm{COCD}_{3}, 500 \mathrm{MHz}\right) \delta: 4.98\left(\mathrm{~d}, J=5.6 \mathrm{~Hz}, 2 \mathrm{H}, \mathrm{CH}_{2}\right)$, 5.09 (s, 2H, $\left.\mathrm{CH}_{2}\right), 6.72(\mathrm{~d}, J=16.0 \mathrm{~Hz}, 1 \mathrm{H}, \operatorname{Ar}(\mathrm{F})-\mathrm{H})$, $6.83 \sim 6.97\left(\mathrm{~m}, 5 \mathrm{H}, \operatorname{Ar}(\mathrm{F})-\mathrm{H}+\mathrm{Ar}\left(\mathrm{OCH}_{2}\right)-\mathrm{H}+\right.$ thiophene$\mathrm{H}+\mathrm{C}=\mathrm{CH}), \quad 7.02 \sim 7.24 \quad(\mathrm{~m}, 9 \mathrm{H}, \quad$ thiophene- $\mathrm{H}+$ $\left.\operatorname{Ar}\left(\mathrm{OCH}_{2}\right)-\mathrm{H}+\mathrm{CH}=+\operatorname{Ar}(\mathrm{F})-\mathrm{H}+\mathrm{Ar}(\mathrm{Cl})-\mathrm{H}\right), 7.26 \sim 7.31$ $(\mathrm{m}, 2 \mathrm{H}, \operatorname{Ar}(\mathrm{Cl})-\mathrm{H}), 7.44 \sim 7.50(\mathrm{~m}, 2 \mathrm{H}$, thiophene- $\mathrm{H}+$ $\left.\operatorname{Ar}\left(\mathrm{OCH}_{2}\right)-\mathrm{H}\right) ;{ }^{13} \mathrm{C} \mathrm{NMR}\left(125 \mathrm{MHz}, \mathrm{CD}_{3} \mathrm{COCD}_{3}\right) \delta: 156.6$, $154.3,141.9,141.0,133.9,132.4,130.5,130.1,129.9$, $129.3,128.2,128.1,128.0,127.8,127.6,127.3,127.1$, $126.4,126.0,125.4,123.2,122.2,121.6,121.4,117.6$, $116.2,114.7,114.6,114.3,114.1,112.8,75.4,69.3$; IR (KBr) v: 3067, 2928, 1595, 1489, 1448, 1238, 1016, 962, 854, 781, 750, 702, $683 \mathrm{~cm}^{-1}$; ESI-MS m/z: $504.2[\mathrm{M}+$ $\mathrm{H}]^{+}$. Anal. calcd for $\mathrm{C}_{29} \mathrm{H}_{23} \mathrm{ClFNO}_{2} \mathrm{~S}$ : C 69.11, H 4.60, N 2.78, found C 69.10, H 4.75, N 2.80.

\section{3 抗 TMV 活性的测试方法}

\subsection{1 活体治疗作用}

采用半叶枯斑法 ${ }^{[15]}$ 选长势一致的心叶烟, 用磷酸 缓冲液将烟草花叶病毒(TMV)粗提液稀释至适宜的浓 度，用毛笔人工摩擦接种于撒有金刚砂的适龄叶片上 (全叶接种病毒, 每叶片人工轻轻涂抹病毒一次, 左右 半叶涂抹力度尽量做到均匀), 接种后用清水冲洗. 待叶 片干后 $(1,3,5,7,9,11 \mathrm{~h}$ 后), 在左半叶涂施化合物溶液, 右半叶涂施对应剂量的溶剂作对照. 随后在光照培养箱 中保湿培养, 控制温度 $(23 \pm 1){ }^{\circ} \mathrm{C}$, 光照 $10000 \mathrm{Lux}, 3$ $4 \mathrm{~d}$ 后观察并记录产生枯斑的数目. 每药剂处理设 3 株, 每株 3 4 片叶. 按上述方法每药剂进行 3 次重复. 


\subsection{2 活体保护作用}

选长势一致的心叶烟, 在左半叶涂施化合物溶液, 右半叶涂施对应剂量的溶剂作对照, 并在光照培养箱中 保湿培养, 控制温度 $(23 \pm 1){ }^{\circ} \mathrm{C}$, 光照 $10000 \mathrm{Lux}, 12 \mathrm{~h}$ 后用磷酸缓冲液将 TMV 病毒粗提液稀释至适宜的浓度, 用毛笔人工摩擦接种于撒有金刚砂的适龄叶片上(全叶 接种病毒, 每叶片人工轻轻涂抹病毒一次, 左右半叶涂 抹力度尽量做到均匀), 接种后用清水冲洗. 随后在光 照培养箱中保湿培养, 控制温度 $(23 \pm 1){ }^{\circ} \mathrm{C}$, 光照 10000 $\mathrm{Lu}, 3 \sim 4 \mathrm{~d}$ 后观察并记录产生枯斑的数目. 每药剂处理 设 3 株, 每株 3 4 片叶. 按上述方法每药剂进行 3 次重 复.

\subsection{3 活体钝化作用}

选长势一致的心叶烟, 用磷酸缓冲液将 $\mathrm{TMV}$ 稀释 至 $6 \times 10^{-3} \mathrm{mg} / \mathrm{mL}$, 将化合物与等体积的病毒汁液混合 钝化 $30 \mathrm{~min}$, 用毛笔人工摩擦接种于撒有金刚砂的适龄 心叶烟左半叶(每叶片人工轻轻涂抹病毒一次, 左右半 叶涂抹力度尽量做判均匀), 对应剂量的溶剂与病毒汁 液混合按种于撒有金刚砂的适龄心叶烟右半叶; 接种后 均用清水冲洗. 随后在光照培养箱中保湿培养, 控制温 度 $(23 \pm 1){ }^{\circ} \mathrm{C}$, 光照 $10000 \mathrm{Lux}, 3 \sim 4 \mathrm{~d}$ 后观察并记录产 生枯斑的数目. 每药剂处理设 3 株, 每株 3 4 片叶. 按 上述方法每药剂进行 3 次重复.

\subsection{4 实验结果的处理}

当空白对照的半叶上呈现明显枯斑, 约在试验 3 $4 \mathrm{~d}$ 后就可调查, 分别记录每片叶的左右半叶的枯斑数, 按下式计算出供试化合物对植物病毒的抑制率, 即相对 效果.

$$
Y=(C-A) / C \times 100 \%
$$

$C$ 为对照组(右半叶)枯斑数, $A$ 为化合物处理组(左半叶) 枯斑数(单位: 个). 每个处理都是用自己的另一半作为 对照, 再设置一组商品宁南霉素的处理作为对比.

辅助材料(Supporting Information) 提供了所合成化 合物的 ${ }^{1} \mathrm{H}$ NMR, ${ }^{13} \mathrm{C}$ NMR, IR 和 ESI-MS 数据. 这些材 料可以免费从本刊网站(http://sioc-journal.cn)上下载.

\section{References}

[1] Kumar, S.; Narain, U.; Tripathi, S.; Misra, K. Bioconjugate Chem. 2001, 12, 464.

[2] Toshiya, M.; Kayo, H.; Ayumi, S. Agric. Food. Chem. 1999, 47, 1.

[3] Ammon, H.; Wahl, M. Planta Med. 1991, 57, 1.

[4] Hahm, E. R.; Cheon, G.; Lee, J.; Kim, B.; Park, C.; Yang, C. H. Cancer Lett. 2002, 184, 89.

[5] Costi, R.; Santo, R. D.; Artico, M.; Massa, S.; Ragno, R.; Loddo, R.; Colla, M. L.; Tramontano, E.; Colla, P. L.; Pani, A. Bioorg. Med. Chem. 2004, 12, 199.

[6] Wang, D. S.; Yang, C. M.; Liu, X. P. J. Nat. Sci. Hunan Normal Univ. 2005, 28, 56 (in Chinese).

(王胜得, 杨春明, 刘兴平, 湖南师范大学自然科学学报, 2005, 28, 56).

[7] Jiang, L.; Wang, H. B.; Mu, W.; Ji, Z. C.; Cao, P. Chin. J. Chem. 2011, 29, 539 .

[8] Yang, S.; Song, B. A.; Liu, X. H. Chin. J. Org. Chem. 2005, 25, 1116 (in Chinese). (杨松, 宋宝安, 刘新华, 有机化学, 2005, 25, 1116.)

[9] Li, S. B.; Hu, D. Y.; Song, B. A.; Yang, S.; Jin, L. H.; Xue, W.; Zeng, S.; Wang, J.; Chen, Z.; Lu, P.; Zhou, X.; Fan, L. E. Chin. J. Org. Chem. 2008, 28, 311 (in Chinese). (李少博，胡德禹，宋宝安，杨松，金林红，薛伟，曾松，王俊， 陈卓, 卢平, 周霞, 杽玲娥, 有机化学, 2008, 28, 311.)

[10] Wang, Z. N.; Hu, D. Y.; Song, B. A.; Yang, S.; Jin, L. H.; Xue, W. Chin. J. Org. Chem. 2009, 29, 1412 (in Chinese).

(王振宁, 胡德禹, 宋宝安, 杨松, 金林红, 薛伟, 有机化学, 2009, 29, 1412.)

[11] Chen, P. L. M.S. Thesis, Guizhou University, Guiyang, 2010 (in Chinese). (陈鹏丽, 硕士论文, 贵州大学, 贵阳, 2010.)

[12] Qiu, Q. J.; Xue, W.; Lu, P.; Wang, Z. C.; Wei, X. Chin. J. Synth. Chem. 2011, 19, 36 (in Chinese). (仇秋娟, 薛伟, 卢平, 王贞超, 魏学, 合成化学, 2011, 19, 36.)

[13] Ma, X. B.; Yin, A. Q.; Xue, S. J. J. Shanghai Normal Univ. (Nat. Sci. 2011, 40, 41.

[14] Sun, G. C.; Chang, J. B.; Chen, R. F.; Xie, J. X.; Cheng, G. F.; Wang, L. F.; He, J.; Wang, L. M. Chin. J. Appl. Chem. 2002, 19, 621 (in Chinese).

(孙纲春, 常俊标, 陈荣锋, 谢晶曦, 程桂芳, 王流芳, 何娟, 王 利敏, 应用化学, 2002, 19, 621.)

[15] Li, S. Z.; Wang, D. H.; Jiao, S. M.; Cheng, S. E. Pesticide Experiment Methods Fungicide Sector, Agriculture Press of China, Beijing, 1991, p. 93 (in Chinese).

(李树正，王笃祜，焦书梅，程书恩，农药实验法：杀菌剂篇，农 业出版社，北京, 1991, p. 93.)

[16] Kong, F. B.; Gao, Y. F.; Chen, X. L.; Li, G. L.; Chen, J. Guangxi Agric. Sci. 2006, 37, 148 (in Chinese).

(孔凡涁, 高扬帆, 陈锡岭, 李广领, 陈军, 广西农业科学, 2006, 37, 148.) 